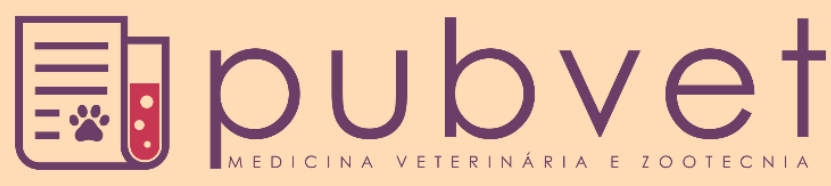

https://doi.org/10.31533/pubvet.v15n12a977.1-10

\title{
Doença inflamatória intestinal: Revisão
}

\author{
Maria Luiza Oliveira Marques ${ }^{1 *} \bullet$, Letícia Duarte Fernandes ${ }^{1 \bullet}$, Natalie Taborda Simone ${ }^{1 \bullet}$, \\ Caio da Silva Caldeira ${ }^{1} \bullet$, William Alves Carneiro Junior ${ }^{1}$
}

${ }^{1}$ Graduando da Universidade Anhembi Morumbi. São Paulo-SP. Brasil.

*Autor para correspondência, E-mail: malumarques14@outlook.com

\begin{abstract}
Resumo. A Doença Inflamatória Intestinal (DII) é uma doença crônica do trato gastrointestinal caracterizada pela manifestação clínica de diarreias, vômitos, perda de apetite e perda de peso progressiva. A doença acomete principalmente cães e gatos de meia idade, sem eleição por gênero. Apesar de possuir uma etiologia pouco elucidada, a maioria dos trabalhos a apresenta como uma doença multifatorial, envolvendo uma resposta imunológica inflamatória exagerada contra bactérias presentes na microbiota intestinal ou antígenos componentes de dietas, a qual o organismo do animal não está adaptado. Algumas raças como Pastor Alemão, West Highland White Terrier, Labrador, Basenji, Shar Pei e Poodle possuem uma predisposição genética à DII, mas sem muito esclarecimento. $\mathrm{O}$ diagnóstico definitivo é estabelecido através da identificação dos sinais crônicos gastrointestinais e da análise, tanto de achados em exames de imagem, como na coleta de material para histopatologia. Alguns dos diagnósticos diferenciais de DII incluem giardíase crônica, linfoma, hipersensibilidade alimentar, infecção por Escherichia coli e insuficiência pancreática exócrina, que são descartados realizando exames complementares. O tratamento para DII envolve, normalmente, alterações no manejo alimentar e uso de medicamentos como antibióticos e fármacos imunossupressores. $\mathrm{O}$ uso de antibióticos justifica-se pelo fato de que antígenos bacterianos podem estar implicados na etiologia da DII, mas também porque frequentemente ocorre desenvolvimento bacteriano secundário. Alguns autores defendem que se deve utilizar a terapêutica dietética exclusiva, tendo em vista que, em certos pacientes, somente o manejo alimentar pode levar à parcial ou completa remissão dos sintomas. Não existem estudos sobre a prevenção da DII, por ser de etiologia genética, mas para o animal que já possui a doença existem protocolos a serem seguidos, como, o uso de rações hipoalergênicas, a administração de prebióticos, probióticos e simbióticos.
\end{abstract}

Palavras-chave: Endoscopia digestiva, infiltrado linfoplasmocitário, trato gastrointestinal

\section{Inflammatory Bowel Disease: Review}

Abstract. Inflammatory Bowel Disease (IBD) denotes a chronic illness of the gastrointestinal tract, characterized by persistent or recurrent clinical signs such as diarrhea, vomiting, loss of appetite and progressive weight loss. The IBD affects mainly middle aged dogs and cats, with no preference for gender. Although not having a clear etiology, most studies present it as a multifactorial disease, involving an exaggerated inflammatory immunological response against microbiota bacterias or antigens in dietary composition, with which the animal organism is not well adapted. The abnormal bacteria growth also seems to be related to IBD development. Specific dog breeds such as German Shepherd, West Highland White Terrier, Labrador, Basenji, Shar Pei and Poodle have a genetic predisposition to IBD, but it is not well elucidated. The diagnosis is established through the identification of the chronic gastrointestinal signs and the analysis both for image findings 
and biopsy histopathology. Some of the differential diagnosis of IBD includes giardiasis, linfoma, dietary hypersensitivity, E. coli infection and exocrine pancreatic insufficiency, that must be discarded with complementary exams. The treatment for IBD normally involves dietary changes and prescribed medications such as antibiotics and immunosuppressive drugs. The use of antibiotics is justified by the fact that bacterial antigens might be implicated in IBD etiology, but also because secondary bacterial development often occurs. Some authors argue the exclusive use of dietary therapy, because in some patients only this change can lead to parcial ou complete remission of the symptoms. There aren't studies about IBD prevention, because of its genetic etiology, but for the animal that has been already diagnosed with the disease there are some protocols to be followed, such as the use of hypoallergenic animal food, prebiotics, probiotics and synbiotics.

Keywords: gastrointestinal tract, lymphoplasmacytic, gastroscopy

\section{Introdução}

A doença inflamatória intestinal (DII) é uma disfunção crônica do trato gastrointestinal que acomete cães e gatos (Jergens et al., 2003), caracterizada pela recorrência de manifestações clínicas que incluem, principalmente, diarreia, vômito, perda de peso e alterações de apetite, sendo confirmada com a presença de infiltrado inflamatório ao exame histológico (Cascon et al., 2017).

As principais raças afetadas, descritas em trabalhos anteriores, são: Pastor Alemão, West Highland White Terrier, Labrador, Basenji, Shar Pei, Golden Retriever, Boxer, Border Collie, Weimaraner e Poodle (Cascon et al., 2017; Craven et al., 2004). A média de idade dos cães descrita por alguns autores varia entre seis e sete anos. Entretanto, estudos recentes incluem animais com menos de 2 anos de idade, o que indica que o diagnóstico diferencial de gastroenterites para esses casos, não deve excluir a DII (Cascon et al., 2017; German et al., 2000; Jergens et al., 1992; Schreiner et al., 2008).

O presente estudo tem como objetivo realizar uma revisão de literatura sobre Doença Inflamatória Intestinal, com ênfase no aspecto diagnóstico da doença, incluindo tanto os achados em exames de imagem, como a coleta de material para análise histopatológica.

\section{Etiopatogenia}

Apesar de sua etiologia pouco conhecida, a Doença Inflamatória Intestinal é uma afecção multifatorial, com a participação de agente infecciosos, mecanismos imunológicos e influências genéticas (Ettinger et al., 2002). O TGI está em constante interação com os microrganismos e, em cães saudáveis, isso ocorre de forma equilibrada e estável, devido ao sistema imunológico (Hall \& German, 2005). Tanto o sistema imunológico inato como o adquirido, têm como objetivo prevenir a interação do antígeno com as células epiteliais, bloqueando uma potencial porta de entrada (Stokes \& Waly, 2006).

Em muitos casos, a DII é desencadeada por uma resposta exagerada ou inapropriada por parte do sistema imunológico contra bactérias e/ou antígenos presentes em uma dieta, no qual o organismo do animal não está corretamente adaptado, a alterações da permeabilidade da mucosa e a micro-organismos intestinais, visto que o cão com DII possui bactérias diferentes das encontradas no cão saudável. Todos esses fatores resultam em alterações do endotélio e microbiota intestinal (Cerquetella et al., 2010; Ettinger et al., 2002; German et al., 2003; Jergens, 1999; Nelson \& Couto, 2015; Slovak et al., 2015; Tams, 2003; Washabau \& Day, 2012).

Devido ao seu alto potencial patogênico, é provável que a microbiota intestinal esteja relacionada com a etiopatogenia da DII. No entanto, este aspecto ainda não foi completamente esclarecido. Em humanos, a doença de Crohn, assim como a colite ulcerativa, afeta as regiões do íleo distal e do cólon, locais onde há maior colonização bacteriana. Além disso, o fato de os antibióticos terem sucesso no tratamento da DII, ressalta a ideia de envolvimento bacteriano (Cave, 2003; Shih \& Targan, 2008). A proliferação acentuada ou a disbiose da microbiota, também podem contribuir à DII (Cave, 2003; Xenoulis et al., 2008). Cave (2003) levantou três hipóteses sobre o papel dos microrganismos entéricos. Eles podem constituir o estímulo primário; podem contribuir para a desregulação qualitativa e 
quantitativa da microbiota; e pode existir uma resposta exagerada à microbiota normal, levando ao agravamento do processo inflamatório (German et al. 2003).

O fator genético é muito presente, considerando que existem algumas raças que carregam uma certa predisposição, como já foi mencionado. Entretanto, não é aprofundado o suficiente para serem implantados programas de melhoramento genético.

Independentemente da causa subjacente, a inflamação dos segmentos intestinais, promove a ruptura das junções estreitas entre as células epiteliais, reduzindo a absorção de sódio e água e promovendo um aumento da permeabilidade intestinal. Esse processo permite que antígenos presentes no lúmen, que normalmente são tolerados (não induzem resposta inflamatória), tenham acesso à mucosa, onde poderá se desenvolver uma reação inflamatória intensa. A resposta imune resultante pode iniciar um ciclo inflamatório. A lesão da mucosa aumenta ainda mais a permeabilidade, o que permite aos antígenos acesso adicional ao seu estrato, amplificando a reação imunológica progressivamente (Ettinger et al., 2017).

As alterações funcionais e estruturais associadas à DII estão relacionadas aos inúmeros mediadores da inflamação, que amplificam o processo. Acredita-se que os metabólitos do ácido araquidônico sejam importantes na patogênese da DII. A via da lipoxigenase leva à produção dos leucotrienos, potentes agentes quimiotáticos, que aumentam a permeabilidade vascular e estimulam a contração da musculatura lisa. A via da cicloxigenase gera prostaglandinas, que também favorecem a permeabilidade vascular, causando vasodilatação e dor (Ettinger et al., 2017).

\section{Manifestações clínicas}

As manifestações clínicas predominantes são diarreia, anorexia e dor à palpação abdominal. A perda de peso progressiva e episódios de êmese ocorrem menos frequentemente, principalmente quando há envolvimento do estômago e/ou intestino delgado. Para serem indicativas de DII, devem se apresentar de forma crônica (Ettinger et al., 2017). Alguns sinais clínicos secundários também podem surgir como presença de muco nas fezes, hematoquezia, tenesmo, borborigmos, flatulência, timpanismo e cólicas.

\section{Diagnóstico}

A Doença Inflamatória Intestinal é um diagnóstico de exclusão, portanto baseia-se em uma alteração histológica inflamatória sem causa notória (Ettinger et al., 2017). Desta forma, o diagnóstico deve seguir alguns estágios. O primeiro é a identificação dos sinais crônicos gastrointestinais, seguido pela triagem e exclusão de outras etiologias que se apresentam com os mesmos sinais clínicos. Por fim, para determinar o diagnóstico definitivo é necessário realizar a análise histopatológica.

Alguns dos principais diagnósticos diferenciais de DII incluem, giardíase crônica, linfoma, hipersensibilidade alimentar, infecção por Escherichia coli e insuficiência pancreática exócrina (Ettinger et al., 2017; Guímaro, 2010; Tams, 2003). Para exclusão dessas etiologias, é sugerido a realização de exames complementares como coproparasitológico seriado, pesquisas de antígenos fecais, análises bioquímicas, mudanças no manejo alimentar, coprocultura, ultrassonografia abdominal, radiografia abdominal e endoscopia digestiva, além da confirmação da desparasitação do animal. Efetuar um perfil hematológico, apesar de não contribuir diretamente para o diagnóstico de DII, pode, não só auxiliar na exclusão de determinadas patologias, como também fornecer indícios de aspectos presentes na patogenia da doença.

Após realizar esta exclusão, deve-se partir aos exames que podem auxiliar ou confirmar o diagnóstico de DII, que envolvem a ultrassonografia abdominal e a endoscopia digestiva alta e baixa com biópsia de diferentes porções do trato gastrointestinal (Suchodolski \& Steiner, 2003).

\section{Ultrassonografia abdominal}

Pela sua acessibilidade, a ultrassonografia abdominal é o exame mais realizado nas suspeitas de infecções do trato gastrointestinal (Figura 1). Apesar de não ser um exame suficiente para estabelecer o diagnóstico definitivo de DII, alguns achados de imagem podem contribuir para a identificação da patologia. Na análise imagiológica deve-se investigar os seguintes aspectos do trato gastrointestinal: espessura (Tabela 1), estratificação e ecogenicidade de túnicas, presença de líquido livre, alta 
motilidade, ecogenicidade peri-intestinal, linfonodomegalia e distribuição focal, multifocal ou difusa da inflamação (Gaschen, 2013).

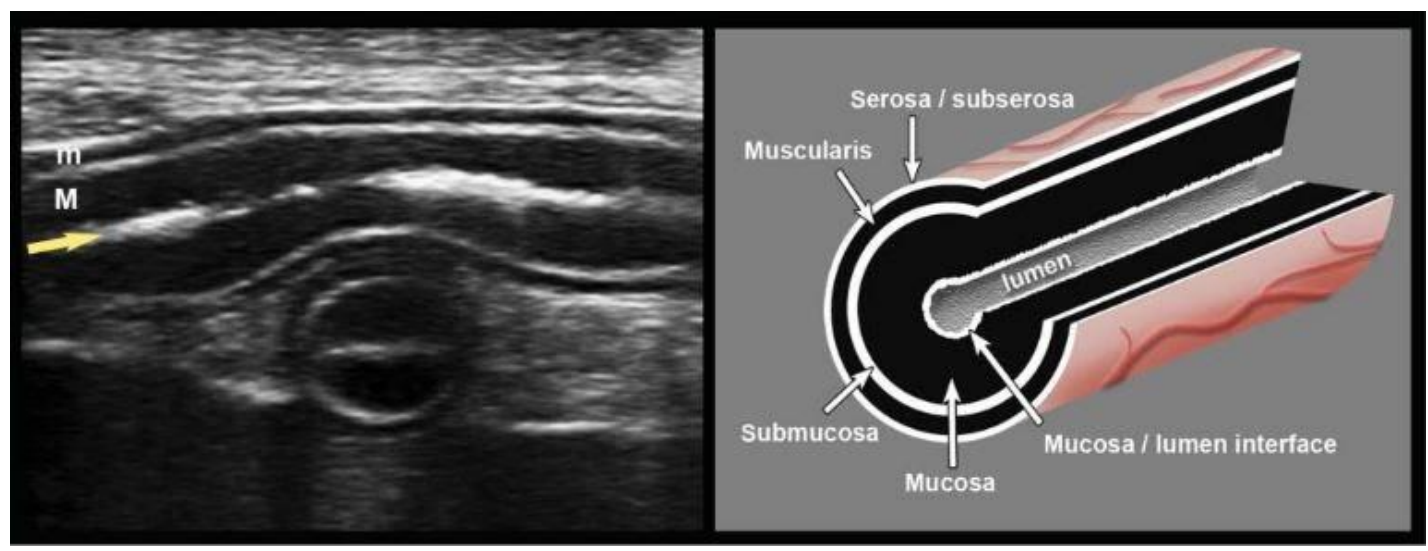

Figura 1. Parênquima intestinal normal. Fonte: Penninck \& D'Anjou (2011).

Tabela 1. Média de valores de referência para espessura de paredes intestinais.

\begin{tabular}{llccccc}
\hline \multirow{2}{*}{ Espécie } & & \multicolumn{5}{c}{ Valores de referência } \\
\cline { 3 - 7 } & \multicolumn{1}{c}{ Estômago } & Duodeno & Jejuno & Ílio & Ceco/Cólon \\
\hline \multirow{2}{*}{ Cães } & $5-30 \mathrm{~kg}$ & $2,5 \mathrm{~mm}$ & $3,8 \mathrm{~mm}$ & $3,0 \mathrm{~mm}$ & $3,0 \mathrm{~mm}$ & $1,5 \mathrm{~mm}$ \\
& $>30 \mathrm{~kg}$ & $2,5 \mathrm{~mm}$ & $4,1 \mathrm{~mm}$ & $3,5 \mathrm{~mm}$ & $3,5 \mathrm{~mm}$ & $1,5 \mathrm{~mm}$ \\
& Gatos & $2,5 \mathrm{~mm}$ & $4,4 \mathrm{~mm}$ & $3,8 \mathrm{~mm}$ & $3,8 \mathrm{~mm}$ & $1,5 \mathrm{~mm}$ \\
\hline
\end{tabular}

Fonte: Adaptado de Penninck \& D’Anjou (2011).

Inflamações intestinais podem aparecer de forma difusa ou focal na imagem ultrassonográfica, causando mudanças na estratificação do tecido (Gaschen et al., 2005; Penninck, 2004; Rudorf et al., 2005). É comum, no processo inflamatório, que haja um aumento na espessura de algumas das túnicas intestinais, principalmente na camada muscular (Penninck, 2002). Em alguns casos mais graves de enterites hemorrágicas, pode haver uma perda da estratificação intestinal (Penninck, 2004). Por fim, algumas manchas focais hiperecóicas podem ser encontradas na mucosa intestinal, sendo bem sugestivas de um quadro de enterite crônica, mas não determinando a causa (Gaschen, 2013). Doenças inflamatórias crônicas podem produzir uma linha fina e hiperecóica adjacente à mucosa, associada a um processo histopatológico de fibrose (Penninck et al., 2010). Os linfonodos jejunais, cólicos, pancreatoduodenais, hepáticos e gástricos drenam todo o segmento intestinal e, durante o exame ultrassonográfico deve ser feita uma varredura de cada um deles. Um linfonodo sem alterações, se apresenta levemente hipoecóico ou isoecóico com o mesentério adjacente (Agthe et al., 2009). Em processos inflamatórios, os linfonodos aumentam de tamanho, mas, geralmente, mantêm sua forma e ecogenicidade (D’Anjou \& Penninck, 2015).

\section{Endoscopia digestiva}

A endoscopia digestiva vem sendo uma importante ferramenta diagnóstica para a avaliação de gastroenterites crônicas e coleta de material para exame histopatológico (Figuras 2 - 3 ), permitindo o diagnóstico de diversas enfermidades (Tams, 2003). Em 1993 num estudo realizado por Münster, o autor concluiu que era possível chegar a um diagnóstico definitivo em $53 \%$ das gastroscopias, $85 \%$ das duodenoscopias e $50 \%$ das colonoscopias. Hoje em dia, é possível chegar a um diagnóstico definitivo em mais de $90 \%$ das endoscopias digestivas altas efetuadas (Tams \& Rawlings, 2010). Além destes valores, segundo Mansell \& Willard (2003), as biópsias obtidas por endoscopia eliminam em 95\% dos casos a necessidade (Guímaro, 2010).

As alterações mais comuns encontradas na endoscopia são o espessamento e o pregueamento da mucosa, eritema, hiperemia, presença de muco, aumento da friabilidade e do aspecto granuloso (Ettinger et al., 2017; Jergens, 1999; Jergens et al., 1992). Todos estes sinais são consistentes com inflamação ou neoplasia e, segundo Tams \& Rawlings (2011), os dois últimos estão comumente associados a DII. A 
aparência da mucosa na DII varia de normal a eritematoso com vários graus de irregularidade. Pode apresentar erosões, ficar mais friável e sangrar ao toque (Tams, 2003). Estas pequenas hemorragias não são normalmente motivo de preocupação (Tams \& Rawlings, 2010).

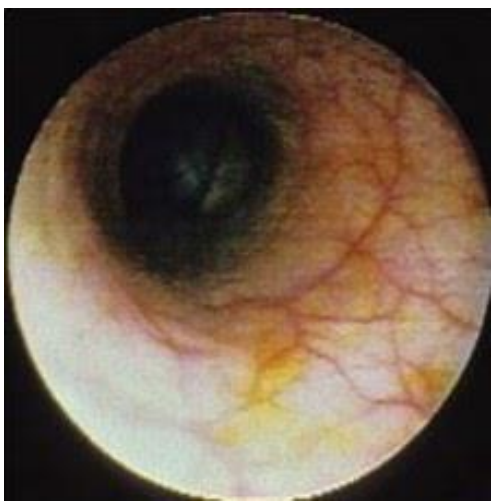

Figura 2. Imagem endoscópica do cólon sem alterações. Fonte: Tams \& Rawlings (2011).

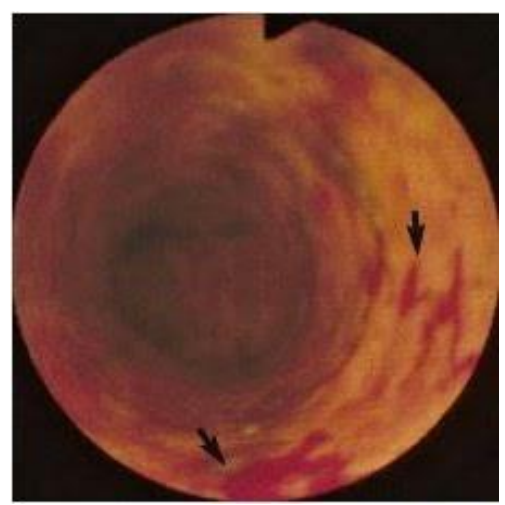

Figura 3. Colite ulcerativa linfoplasmocitária com úlceras hemorrágicas (setas). Fonte: Tams \& Rawlings (2011).

\section{Análise histopatológica}

O exame histológico é considerado padrão ouro para o diagnóstico de Doença Inflamatória Intestinal (Jergens, 2004; Münster et al., 2006). A infiltração da mucosa intestinal pode ser classificada de acordo com as células presentes com maior predominância na mucosa intestinal. $\mathrm{O}$ infiltrado mais comum em pequenos animais é o linfoplasmocitário, mas também podem observar-se outros tipos como eosinofílico, granulomatoso e neutrofílico (Cerquetella et al., 2010; Day et al., 2008; Ettinger et al., 2017; Evans et al., 2006; Reilly, 2006).

\section{Infiltrado linfoplasmocitário}

A enterite linfoplasmocitária é a mais frequentemente descrita em pequenos animais, sendo caracterizada por um infiltrado rico em linfócitos e plasmócitos (Figura 4) na lâmina própria que pode estender-se até à submucosa e muscular, associada a modificações na arquitetura da mucosa (GarcíaSancho et al., 2011; Gaschen, 2011; Hall \& German, 2008; Maeda et al., 2012; Saunders, 2007).
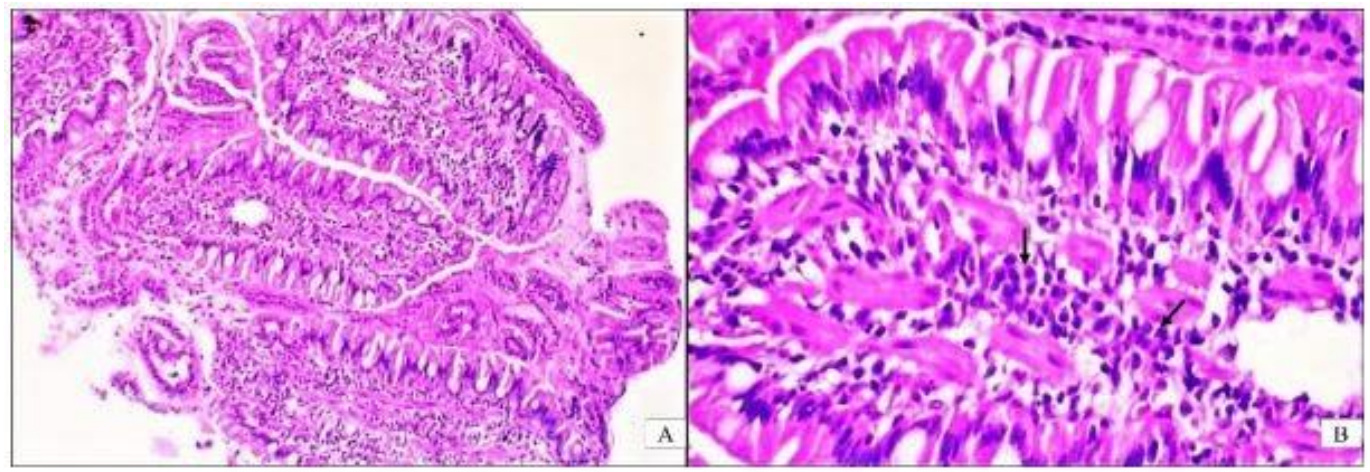

Figura 4. Infiltrado linfoplasmocitário em duodeno. A) Corte de região duodenal; B) Aumento do corte obtido em A As setas indicam a presença de infiltrado linfoplasmocitário. Fonte: Gouvêa et al. (2020).

\section{Infiltrado eosinofílico}

É o segundo tipo mais frequentemente encontrado no cão, apesar de ser uma forma bastante mais rara que a linfoplasmocítica (Ettinger et al., 2017; German et al., 2002; Saunders, 2007; Tams, 2003). Normalmente além do intestino delgado, existe envolvimento do estômago e/ou cólon, ou seja, gastroenterocolite eosinofílica (Ettinger et al., 2017; Tams, 2003).

A presença moderada a grave de eosinófilos na amostra de biópsia intestinal acompanhada de eosinófilos no sangue periférico em circulação, sugere possível infestação parasitária ou intolerância à 
dieta, sendo que a presença de infiltrado de eosinófilos na lâmina própria não é patognomônico de DII eosinofílica. Este tipo de inflamação pode estar também associado a hipoadrenocorticismo, alergias cutâneas, neoplasias ou doenças do foro respiratório (German, 2005; Hall \& German, 2010; Sherding, 2003; Simpson \& Jergens, 2011).

\section{Infiltrado granulomatoso}

A gastroenterite granulomatosa é pouco frequente no cão (German, 2009) e caracterizada por um infiltrado com abundância de macrófagos, originando a formação de granulomas, sendo que em gatos

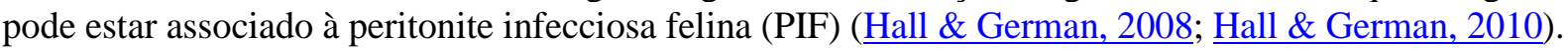

\section{Infiltrado neutrofílico}

A infiltração neutrofílica também é rara, estando em muitas situações descrita como sendo um infiltrado celular misto (German, 2009). Os neutrófilos constituem uma componente menor da resposta inflamatória na IBD e são sobretudo vistos em lesões erosivas do TGI. As amostras histológicas mostram densos infiltrados de linfócitos, plasmócitos e neutrófilos. Tem vindo a ser associada a Clostridium spp e Campylobacter jejuni (Cave, 2003; Jergens, 1999; Sherding, 2003).

As alterações epiteliais e da lâmina própria têm vindo a ganhar uma importância crescente, quando avaliado o aumento da celularidade leucocitária na IBD. Por isso, os sistemas de classificação mais recentemente criados têm tido em conta não só o aumento na população de leucócitos como também as alterações associadas. O critério mais utilizado é o aumento do número de leucócitos na lâmina própria (Brown et al., 2007; Yamasaki et al. 1996).

\section{Perfil hematológico}

Não existe nenhuma alteração sanguínea indicativa de DII. Entretanto, alguns estudos mencionam alterações que ocasionalmente aparecem no hemograma. Pode ser observada neutrofilia com ou sem desvio à esquerda devido à inflamação crónica (Hall \& German, 2005). A monocitose e a linfopenia não são incomuns (Hall, 1999; Jacobs et al., 1990; Münster, 1995; Tams, 2003). A perda de sangue intestinal crônica na DII também pode provocar anemia por deficiência em ferro (Ristic \& Stidworthy, 2002).

\section{Tratamento}

Independentemente do tipo de infiltrado inflamatório, o tratamento envolve normalmente alterações no manejo alimentar e uso de medicamentos como antibióticos e fármacos imunossupressores (Hall \& German, 2005).

Em alguns pacientes o manejo alimentar pode levar à parcial ou completa remissão dos sintomas. Por esta razão, alguns autores preconizam que deve experimentar-se a terapêutica dietética sozinha (Hall \& German, 2005). Os benefícios do tratamento dietético são a melhoria clínica, a redução da hipersensibilidade aos antígenos dietéticos, a alteração da motilidade intestinal e ainda os efeitos na composição da microbiota intestinal, na morfologia e na função da mucosa (Sherding, 2003; Tams, $\underline{2003}$ ). Nos casos em que ocorre anorexia periódica e em que existem déficits nutricionais graves, pode ser necessário a colocação de um tubo de alimentação por um curto período, mas apenas se o animal tiver o TGI funcional (Matz \& Guilford, 2003).

O uso de antibióticos justifica-se pelo fato de que antígenos bacterianos podem estar implicados na etiologia da DII (German, 2009), mas também porque frequentemente ocorre desenvolvimento bacteriano secundário à DII. O principal antibiótico utilizado é o metronidazol, possui características imunomoduladoras pela inibição da imunidade celular, tem um bom espectro contra bactérias anaeróbias (Allen et al., 2005; Tennant, 2005). A dose utilizada é de 7,5 a $15 \mathrm{mg} / \mathrm{kg}$ cada 12 horas. Só quando não surgem melhorias com a alteração dietética e antibióticos é que se utilizam medicamentos imunossupressores (Hall \& German, 2005).

Os corticosteroides são os medicamentos de eleição na DII, sendo a prednisolona a mais utilizada (Jergens, 2002; Jergens, 2007). Além das suas propriedades anti-inflamatórias e imunossupressoras atua ainda na estimulação do apetite, aumento da absorção intestinal de água, sódio e glutamina. A sua dose 
inicial deve ser escolhida de acordo com a gravidade da inflamação, sendo habitualmente de 1 a $2 \mathrm{mg} / \mathrm{kg}$ VO a cada 12 horas durante 2 a 4 semanas. Em seguida faz-se uma redução gradual que pode prolongarse por semanas a meses (Hall \& German, 2005; Jergens, 2002).

A budesonida é um glicocorticoide que constitui uma alternativa à DII. É utilizado em casos não responsivos à terapêutica conjunta com prednisolona, metronidazol e manejo alimentar (German, 2006). A dose utilizada é de 1 a $3 \mathrm{mg} / \mathrm{animal}$ a cada 12 horas.

Alguns cães com DII apresentam desidratação que pode resultar das perdas por vômito ou por diarreia, mas também pode ser devido à diminuição da ingestão de água. Se o animal apresentar sinais de desidratação é necessária a administração de fluidoterapia por via intravenosa (Guímaro, 2010).

O segundo sinal clínico mais frequente na DII é o vómito. O uso de anti-eméticos é sugerido nos pacientes em que o vômito seja agudo ou que provoque desconforto ou desequilíbrios hidroeletrolíticos.

\section{Prevenção}

Por ser principalmente de etiologia genética, não existem estudos sobre a prevenção da DII. Para o animal que já possui a doença, alguns protocolos podem ser seguidos para prevenir a recorrência dos sinais clínicos. O uso de rações hipoalergênicas, a administração de prebióticos, probióticos e simbióticos podem contribuir para que a doença se mantenha silenciosa.

O tutor deve estar ciente que se trata de uma doença crônica e que por isso o objetivo do tratamento é conseguir o controle dos sintomas e evitar que surjam recorrências (Elwood, 1999).

\section{Considerações finais}

A Doença Inflamatória Intestinal é uma patologia pouco diagnosticada e que frequentemente passa despercebida pelos clínicos. Caso haja suspeita, é imprescindível realizar a exclusão de outras doenças e utilizar-se do histórico do animal, assim como exames complementares que auxiliem no diagnóstico. A biópsia com análise histopatológica ainda é o único exame definitivo para confirmar a suspeita clínica, encontrando, na maioria das vezes, um infiltrado do tipo linfoplasmocitário. Uma vez diagnosticada, o tratamento deve ser iniciado com uso de antibióticos, corticoides, fármacos imunossupressores e alterações no manejo alimentar. Por fim, realizar um acompanhamento periódico do animal é fundamental para avaliar a evolução e melhora do paciente.

\section{Referências bibliográficas}

Agthe, P., Caine, A. R., Posch, B., \& Herrtage, M. E. (2009). Ultrasonographic appearance of jejunal lymph nodes in dogs without clinical signs of gastrointestinal disease. Veterinary Radiology \& Ultrasound, 50(2), 195-200. https://doi.org/10.1111/j.1740-8261.2009.01516.x.

Allen, D.G., Dowling, P.M., Smith, D.A., Pasloske, K. \& Woods, J.P. 2005. Handbook of Veterinary Drugs.

Brown, C.C., Baker, D.C. \& Barker, I.K. 2007. Alimentary and peritoneum: alimentary system. In Jubb, Kennedy \& Palmer's, Pathology of Domestic Animals (5th ed.).

Cascon, C. M., Mello, M. F. V, Leite, J. S., \& Ferreira, A. M. R. (2017). Avaliação clínica, endoscópica e histopatológica de cães com doença inflamatória intestinal. Pesquisa Veterinária Brasileira, 37, 1287-1291. https://doi.org/10.1590/S0100-736X2017001100015.

Cave, N. J. (2003). Chronic inflammatory disorders of the gastrointestinal tract of companion animals. New Zealand Veterinary Journal, 51(6), 262-274. https://doi.org/10.1080/00480169.2003.36380.

Cerquetella, M., Spaterna, A., Laus, F., Tesei, B., Rossi, G., Antonelli, E., Villanacci, V., \& Bassotti, G. (2010). Inflammatory bowel disease in the dog: differences and similarities with humans. World Journal of Gastroenterology, 16(9), 1050. https://doi.org/10.3748/wjg.v16.i9.1050.

Craven, M., Simpson, J. W., Ridyard, A. E., \& Chandler, M. L. (2004). Canine inflammatory bowel disease: retrospective analysis of diagnosis and outcome in 80 cases (1995-2002). Journal of Small Animal Practice, 45(7), 336-342. https://doi.org/10.1111/j.1748-5827.2004.tb00245.x.

D’Anjou, M. A., \& Penninck, D. (2015). Atlas of Small Animal Ultrasonography. In D. Penninck \& M. A. D’Anjou (Eds.), Liver (Issue 2, p. 223). Wiley Blackwell. 
Day, M. J., Bilzer, T., Mansell, J., Wilcock, B., Hall, E. J., Jergens, A., Minami, T., Willard, M., \& Washabau, R. (2008). Histopathological standards for the diagnosis of gastrointestinal inflammation in endoscopic biopsy samples from the dog and cat: a report from the World Small Animal Veterinary Association Gastrointestinal Standardization Group. Journal of Comparative Pathology, 138, S1-S43.

Elwood, C.M. (1999). Diarrhoea. In J.K. Dunn, Textbook of Small Animal Medicine.

Ettinger, S. J., Fedlman, E. C., \& Taibo, R. A. (2002). Tratado de medicina interna veterinaria: enfermedades del perro y el gato. Manole.

Ettinger, S. J., Feldman, E. C., \& Cote, E. (2017). Textbook of Veterinary Internal Medicine-eBook. Elsevier Health Sciences.

Evans, S. E., Bonczynski, J. J., Broussard, J. D., Han, E., \& Baer, K. E. (2006). Comparison of endoscopic and full-thickness biopsy specimens for diagnosis of inflammatory bowel disease and alimentary tract lymphoma in cats. Journal of the American Veterinary Medical Association, 229(9), 1447-1450. https://doi.org/10.2460/javma.229.9.1447 .

García-Sancho, M., Sainz, Á., Villaescusa, A., Rodríguez, A., \& Rodríguez-Franco, F. (2011). White spots on the mucosal surface of the duodenum in dogs with lymphocytic plasmacytic enteritis. Journal of Veterinary Science, 12(2), 165-169. https://doi.org/10.4142/jvs.2011.12.2.165.

Gaschen, L. (2013). Ultrassonographic Imaging of the gastrointestinal tract. In R.J. Washabau \& M. J. Day (Eds.), Diagnostic Imaging of gastrointestinal tract (pp. 228-235). Elsevier.

Gaschen, L. (2011). Ultrasonography of small intestinal inflammatory and neoplastic diseases in dogs and cats. The Veterinary Clinics of North America. Small Animal Practice, 41(2), 329-344. https://doi.org/10.1016/j.cvsm.2011.01.002.

Gaschen, L., Kircher, P., Lang, J., Gaschen, F., Allenspach, K., \& Gröne, A. (2005). Pattern recognition and feature extraction of canine celiac and cranial mesenteric arterial waveforms: normal versus chronic enteropathy-a pilot study. The Veterinary Journal, 169(2), 242-250. https://doi.org/10.1016/j.tvj1.2004.01.028.

German, A. J., Hall, E. J., \& Day, M. J. (2000). Relative deficiency in IgA production by duodenal explants from German shepherd dogs with small intestinal disease. Veterinary Immunology and Immunopathology, 76(1-2), 25-43. https://doi.org/10.1016/s0165-2427(00)00191-4 .

German, A. J., Hall, E. J., \& Day, M. J. (2003). Chronic intestinal inflammation and intestinal disease in dogs. Journal of Veterinary Internal Medicine, 17(1), 8-20. https://doi.org/10.1111/j.1939-1676.2003.tb01318.x.

German, A. J., Holden, D. J., Hall, E. J., \& Day, M. J. (2002). Eosinophilic diseases in two Cavalier King Charles spaniels. Journal of Small Animal Practice, 43(12), 533-538. https://doi.org/10.1111/j.1748-5827.2002.tb00026.x.

German, A.J. 2005. Diseases of the small intestine. In: E.J. Hall, J.W. Simpson \& D.A. Williams (Eds.), BSAVA Manual of Canine and Feline Gastroenterology (2. $\left.{ }^{\mathrm{a}} \mathrm{Ed}\right)$.

German A.J. 2006. Update on inflammatory bowel disease. Congress of the World Small Animal Veterinary Association, 31 Prague, Czech Republic.

German, A.J. 2009. Gastrointestinal diseases: inflammatory bowel disease. In J.D. Bonagura \& D.C. Twedt (eds.), Kirk's Current Veterinary Therapy, (14th Ed.).

Gouvêa, F. N., Pennachi, C. S., Assaf, N. D., Arantes, E. A. L., Stefaniszen, A. G., Vieira, E. M., Genari, V., Guimarães-Okamoto, P. T. C., \& Melchert, A. (2020). Doenças inflamatória intestinal em cães Relato de casos. Ars Veterinaria, 36(4), 332-336. https://doi.org/10.15361/21750106.2020v36n4p332-336.

Guímaro, J. O. M. (2010). Doença inflamatória crónica do intestino: estudo comparativo entre a imagem endoscópica e o resultado histopatológico em 73 canídeos. Universidade Técnica de Lisboa.

Hall, E.J. (1999). Clinical laboratory evaluation of small intestinal function. Veterinary Clinics of North America: Small Animal Practice, 29, 441-469. Doi https://doi.org/10.1016/S0195-5616(99)50030-2.

Hall, E.J., \& German, A.J. 2005. Gastrointestinal disease: diseases of the small intestine. In S.J. Ettinger \& E.C. Feldman (Eds.), Textbook of Veterinary Internal Medicine (6th ed.).

Hall, E.J. \& German, A.J. 2010. Diseases of the small intestine. In: S.J. Ettinger \& E.C. Feldman (Eds.), Textbook of Veterinary Internal Medicine (7. $\left.{ }^{\mathrm{a}} \mathrm{Ed}.\right)$. 
Hall, E. J., \& German, A. J. (2008). Inflammatory bowel disease. In J. M. Steiner (Ed.), Small animal gastroenterology.

Jacobs, G., Collins-Kelly, L., Lappin, M. \& Tyler, D. (1990). Lymphocytic-plasmacytic enteritis in 24 dogs. Journal of Veterinary Internal Medicine, 4, 45-53. Doi https://doi.org/10.1111/j.1939-1676.1990.tb03102.x.

Jergens, A E. (1999). Inflammatory bowel disease: current perspectives. Veterinary Clinics of North America: Small Animal Practice, 29(2), 501-521. https://doi.org/10.1016/S0195-5616(99)50032-6.

Jergens, A E, Moore, F. M., Haynes, J. S., \& Miles, K. G. (1992). Idiopathic inflammatory bowel disease in dogs and cats: 84 cases (1987-1990). Journal of the American Veterinary Medical Association, 201(10), 1603-1608.

Jergens, A.E. 2007. Current and emerging options for IBD therapy. European College of Veterinary Internal Medicine for Companion Animals Congress, 17 Budapest, Hungary. Budapest: ECVIM-CA.

Jergens, A. E. (2004). Clinical assessment of disease activity for canine inflammatory bowel disease. Journal of the American Animal Hospital Association, 40(6), 437-445. https://doi.org/10.5326/0400437.

Jergens, Albert E, Schreiner, C. A., Frank, D. E., Niyo, Y., Ahrens, F. E., Eckersall, P. D., Benson, T. J., \& Evans, R. (2003). A scoring index for disease activity in canine inflammatory bowel disease. Journal of Veterinary Internal Medicine, 17(3), 291-297. https://doi.org/10.1892/08916640(2003)017<0291:ASIFDA>2.3.CO;2.

Jergens, A. E. (2002). Inflammatory bowel disease in the dog and cat. World Small Animal Veterinary Association Congress, 27, Granada, Spain. Granada: AVEPA.

Knudsen, C. S., Arthurs, G. I., Hayes, G. M., \& Langley-Hobbs, S. J. (2012). Long bone fracture as a complication following external skeletal fixation: 11 cases. Journal of Small Animal Practice, 53(12), 687-692. https://doi.org/10.1111/j.1748-5827.2004.tb00245.x.

Maeda, S., Ohno, K., Nakamura, K., Uchida, K., Nakashima, K., Fukushima, K., Tsukamoto, A., GotoKoshino, Y., Fujino, Y., \& Tsujimoto, H. (2012). Mucosal imbalance of interleukin-1 $\beta$ and interleukin-1 receptor antagonist in canine inflammatory bowel disease. The Veterinary Journal, 194(1), 66-70. https://doi.org/10.1016/j.tvj1.2012.02.026.

Mansell, J., \& Willard, M. D. (2003). Biopsy of the gastrointestinal tract. Veterinary Clinics: Small Animal Practice, 33(5), 1099-1116. https://doi.org/10.1016/S0195-5616(03)00056-1.

Matz, M.E. \& Guilford, W.G. (2003). Laboratory procedures for the diagnosis of gastrointestinal tract diseases of dogs and cats. New Zealand Veterinary Journal, 51, 292-301. Doi https://doi.org/10.1080/00480169.2003.36383

Münster, M. (1995). Die lympho-plasmazelluläre Enteritis bei Hund und Katze - klinische Befunde und Therapieergebnisse. Kleintierpraxis, 40, 581-590.

Münster, M. (1993). Effizienz der Endoskopie bei Magen-Darm-Erkrankungen von Hund und Katze. Der praktische Tierarzt, 4, 309-312.

Münster, M., Hörauf, A., \& Bilzer, T. (2006). Bestimmung des Krankheitsschweregrades und des Ergebnisses diätetischer, antibiotischer und immunsuppressiver Interventionen durch Anwendung des caninen IBD Aktivitätsindex bei 21 Hunden mit chronisch entzündlicher Darmkrankheit. Berliner Und Münchener Tierärztliche Wochenschrift, 119, 493-505.

Nelson, R. W., \& Couto, C. G. (2015). Medicina interna de pequenos animais (Issue 1). Elsevier Editora.

Penninck, D.G. (2004). Trato gastrointestinal. In: Nyland, T. \& Matton, J. S. (Eds.), Ultrassom diagnóstico em pequenos animais (pp. 211-234). Roca, Brasil.

Penninck, D G. (2002). Gastrointestinal tract. In T. G. Nyland \& J. S. Mattoon (Eds.), Small animal diagnostic ultrasound. Saunders Elsevier.

Penninck, D G, \& D’Anjou, M. A. (2011). Atlas de ultrassonografia de Pequenos animais (p. 513p.). Guanabara Koogan.

Penninck, Dominique G, Webster, C. R. L., \& Keating, J. H. (2010). The sonographic appearance of intestinal mucosal fibrosis in cats. Veterinary Radiology \& Ultrasound, 51(4), 458-461. https://doi.org/10.1111/j.1740-8261.2010.01679.x. 
Reilly, C. (2006). BSAVA Manual of canine and feline gastroenterology. Journal of Small Animal Practice, 47(4), 228.

Ristic, J.M.E. \& Stidworthy M.F. (2002). Two cases of severe iron-deficiency anaemia due to inflammatory bowel disease in the dog. Journal of Small Animal Practice,43, 8083. Doi https://doi.org/10.1111/j.1748-5827.2002.tb00034.x

Rudorf, H., Van Schaik, G., O’Brien, R. T., Brown, P. J., Barr, F. J., \& Hall, E. J. (2005). Ultrasonographic evaluation of the thickness of the small intestinal wall in dogs with inflammatory bowel disease. Journal of Small Animal Practice, 46(7), 322-326. https://doi.org/10.1111/j.1748-5827.2005.tb00327.x.

Saunders, W. B. (2007). Kirk's Current Veterinary Therapy.

Schreiner, N. M. S., Gaschen, F., Gröne, A., Sauter, S. N., \& Allenspach, K. (2008). Clinical signs, histology, and CD3-positive cells before and after treatment of dogs with chronic enteropathies. Journal of Veterinary Internal Medicine, 22(5), 1079-1083. https://doi.org/10.1111/j.1939-1676.2008.0153.x.

Sherding, R.G. (2003). Diseases of the large intestine. In T.R. Tams, Handbook of Small Animal Gastroenterology, (2nd ed.).

Shih, D. Q., \& Targan, S. R. (2008). Immunopathogenesis of inflammatory bowel disease. World Journal of Gastroenterology, 14(3), 390. https://doi.org/10.3748\%2Fwjg.14.390.

Simpson, K.W., \& Jergens, A.E. (2011). Pitfalls and progress in the diagnosis and management of canine inflammatory bowel disease. Veterinary Clinics of North America: Small Animal Practice, 41, 381398. Doi https://doi.org/10.1016/j.cvsm.2011.02.003.

Slovak, J. E., Wang, C., Sun, Y., Otoni, C., Morrison, J., Deitz, K., LeVine, D., \& Jergens, A. E. (2015). Development and validation of an endoscopic activity score for canine inflammatory bowel disease. The Veterinary Journal, 203(3), 290-295. https://doi.org/10.1016/j.tvj1.2014.12.030.

Stokes, C., \& Waly, N. (2006). Mucosal defence along the gastrointestinal tract of cats and dogs. Veterinary Research, 37(3), 281-293. https://doi.org/10.1051/vetres:2006015.

Suchodolski, J. S., \& Steiner, J. M. (2003). Laboratory assessment of gastrointestinal function. Clinical Techniques in Small Animal Practice, 18(4), 203-210. https://doi.org/10.1016/s1096-2867(03)00075-6.

Tams, T R. (2003). Handbook of small animal gastroenterology. Elsevier Health Sciences.

Tams, T R, \& Rawlings, C. A. (2011). Small animal endoscopy. Elsevier Mosby. https://doi.org/10.1016/C2009-0-52089-2.

Tams, Todd R, \& Rawlings, C. A. (2010). Small animal endoscopy-E-Book. Elsevier Health Sciences.

Tennant, B. (Ed.).2005. BSAVA Small Animal Formular (5th ed.).

Washabau, Robert J, \& Day, M. J. (2012). Canine and Feline Gastroenterology-E-Book. Saunders Company.

Xenoulis, P. G., Palculict, B., Allenspach, K., Steiner, J. M., Van House, A. M., \& Suchodolski, J. S. (2008). Molecular-phylogenetic characterization of microbial communities imbalances in the small intestine of dogs with inflammatory bowel disease. FEMS Microbiology Ecology, 66(3), 579-589. https://doi.org/10.1111/j.1574-6941.2008.00556.x.

Yamasaki, K., Suematsu, H. \& Takahashi, T. (1996). Comparison of gastric and duodenal lesions in dogs and cats with and without lymphocytic-plasmacytic enteritis. Journal of the American Veterinary Medical Association, 209, 95-97.

Histórico do artigo

Recebido: 8 de agosto de 2021

Aprovado: 21 de setembro de 2021

Disponível online: 6 de novembro de 2021
Licenciamento: Este artigo é publicado na modalidade Acesso Aberto sob a licença Creative Commons Atribuição 4.0 (CC-BY 4.0), a qual permite uso irrestrito, distribuição, reprodução em qualquer meio, desde que $\mathrm{o}$ autor e a fonte sejam devidamente creditados. 